\title{
Dinoflagelados (Dinophyta) de los órdenes Prorocentrales y Dinophysiales del Sistema Arrecifal Veracruzano, México
}

\author{
Dulce Parra-Toriz ${ }^{1,3}$, María de Lourdes Araceli Ramírez-Rodríguez ${ }^{1}$ \& \\ David Uriel Hernández-Becerril ${ }^{2}$ \\ 1. Facultad de Biología, Universidad Veracruzana, Circuito Gonzalo Beltrán s/n, Zona Universitaria, Xalapa, Veracruz, \\ 91090 México; marilu513@hotmail.com \\ 2. Instituto de Ciencias del Mar y Limnología, Universidad Nacional Autónoma de México (UNAM). Apartado Postal \\ 70-305, México D.F. 04510 México; dhernand@cmarl.unam.mx \\ 3. Posgrado en Ciencias del Mar. Instituto de Ciencias del Mar y Limnología, Universidad Nacional Autónoma de \\ México (UNAM). Apartado Postal 70-305, México D.F. 04510 México; parratoriz031184@gmail.com
}

Recibido 12-III-2010. C Corregido 24-VIII-2010. Aceptado 23-IX-2010.

\begin{abstract}
Dinoflagellates (Dinophyta) of orders Dinophysiales and Prorocentrales of the Veracruz Reef System, Mexico. Dinoflagellates are a major taxonomic group in marine phytoplankton communities in terms of diversity and biomass. Some species are also important because they form blooms and/or produce toxins that may cause diverse problems. The composition of planktonic dinoflagellates of the orders Prorocentrales and Dinophysiales, in the Veracruz Reef System, were obtained during the period of October 2006 to January 2007. For this, samples were taken from the surface at 10 stations with net of $30 \mu \mathrm{m}$ mesh, and were analyzed by light and scanning electron microscopy. Each species was described and illustrated, measured and their distribution and ecological data is also given. A total of nine species were found and identified, belonging to four genera: Dinophysis was represented by three species; Prorocentrum by three, Phalacroma by two, and only one species of Ornithocercus was detected. From the samples, four potentially toxin-producer species were found: Dinophysis caudata, D. rapa, Phalacroma rotundata and Prorocentrum micans. The number of species found in this study is low, especially considering the higher numbers observed in other areas of the Gulf of Mexico, where some reports have recorded up to 53 species of the order Dinophysiales and 14 for Prorocentrales. Identification keys for orders, genera and species for the study area are provided with this study. Rev. Biol. Trop. 59 (1): 501514. Epub 2011 March 01.
\end{abstract}

Key words: dinoflagellates, morphology, phytoplankton, Veracruz Reef System, taxonomy.

Los dinoflagelados son considerados como el grupo más abundante y diverso de microalgas del fitoplancton después de las diatomeas. La talla de estos organismos va de $5 \mu \mathrm{m}$ hasta $2 \mathrm{~mm}$ y en ocasiones desarrollan estructuras anexas como aletas, cuernos o espinas. Algunos dinoflagelados se encuentran provistos de una cubierta rígida llamada teca y se conocen como dinoflagelados tecados o armados.

Entre las especies que se han reportado como productoras de toxinas en México se mencionan a Gymnodinium catenatum Graham y Karenia brevis (Davis) Hensen et Moestrup, son atecadas o desnudas, y Pyrodinium bahamense var. compressum (Böhm) Steidinger, Tester et Taylor que es una especie tecada y las potencialmente nocivas de los géneros Alexandrium, Prorocentrum, Dinophysis y Phalacroma (Hernández-Becerril et al. 2007). Estos tres últimos géneros pertenecientes a los órdenes Prorocentrales y Dinophysiales están relacionados con la producción de toxinas causantes de envenenamiento diarreico por consumo de mariscos, DSP (Diarrhetic 
Shellfish Poisoning) (Hernández-Becerril et al. 2007).

Los miembros del orden Prorocentrales, se caracterizan por poseer una teca sencilla, bivalvar (derecha e izquierda), cada valva esta formada por una sola placa que suele estar perforada por poros generalmente oblicuos, a veces densos y que con frecuencia se disponen en campos o hileras bien definidas en la región oral a veces una de las placas se prolonga hacia adelante en un apéndice apical agudo triangular o espiniforme (espina apical) poco o muy desarrollado, por la inserción flagelar anterior y la reducción en número y tamaño de placas ( 8 a 9) en esa zona (Dodge \& Bibby 1973, Balech 1988).

El orden de los Dinophysiales incluye diversas formas marinas, móviles y tecadas, aplanadas lateralmente, la superficie de la teca generalmente presenta poros, poseen aletas cingulares y sulcales con un desarrollo variable y una sutura sagital aserrada extendida a todo lo largo de su cuerpo, así la teca presenta dos valvas: izquierda y derecha, la aleta sulcal izquierda está bien desarrollada y provista de tres radios que la sostienen R1, R2 y R3, y tienen en general un número de placas estable, 18 placas (con ciertas excepciones): 2 apicales, 4 epitecales, 4 cingulares, 4 sulcales y 4 hipotecales (Sournia 1986, Hernández-Becerril et al. 2003, 2008).

El propósito de este trabajo es determinar la composición específica y variación temporal de las especies de dinoflagelados de los órdenes Prorocentrales y Dinophysiales del Parque Nacional Sistema Arrecifal Veracruzano (PSAV). Recientemente en dicha zona se ha dado a conocer la composición de dinoflagelados tóxicos o potencialmente tóxicos (Licea et al. 2004), así como dinoflagelados bentónicos y especies planctónicas del género Protoperidinium (Okolodkov et al. 2007, Okolodkov 2008).

\section{MATERIALES Y MÉTODOS}

En el suroeste del Golfo de México se han reportado 46 arrecifes de coral. De estos 31 pertenecen al Sistema Arrecifal Veracruzano (SAV). El SAV se localiza frente al puerto de
Veracruz y la población de Antón Lizardo: enlaza al NW con el SAV Norte, situado frente a la laguna de Tamiahua y al este con el complejo arrecifal de Campeche y Yucatán. Los arrecifes que componen el SAV se encuentran delimitados por los ríos La Antigua al norte y Papaloapan al sur. La desembocadura del río Jamapa divide al sistema en dos áreas, una frente al puerto de Veracruz y otra frente al poblado de Antón Lizardo; esto provoca que las aguas circundantes sean turbias y poco transparentes. La temperatura promedio anual en la zona arrecifal es de $26.1{ }^{\circ} \mathrm{C}$, el clima de la zona de estudio se define como caliente-húmedo con lluvias en verano y su esquema climático puede sintetizarse en dos épocas del año 1) La época de nortes que abarca desde Septiembre hasta Abril, con escasa precipitación, temperatura baja y frecuentes invasiones de masas de aire frío del Norte. 2) La época de lluvias de Mayo a Agosto, que es un periodo cálido, caracterizado por temperatura elevada, alta precipitación entre Junio y Agosto y vientos débiles del este que soplan más o menos permanentemente (Gutiérrez et al. 1993, Tunnell et al. 2007, Vargas-Hernández et al.1993).

Durante el periodo comprendido entre octubre de 2006 y enero de 2007, se recolectaron mensualmente muestras de fitoplancton por medio de una red para fitoplancton de $30 \mu \mathrm{m}$ de luz de malla, en 10 sitios de muestreo distribuidos en el SAV (Fig. 1). En cada estación se realizaron arrastres horizontales superficiales $(30 \mathrm{~cm})$ con una duración aproximada de cinco minutos, a una velocidad entre los dos y tres nudos. Las muestras obtenidas se fijaron con una solución de formaldehído al 4\%.

La determinación de las especies se hizo mediante el método de Microscopía de Luz (ML), empleando un microscopio Leica CME triocular 11502 y un microscopio Olympus BX40, acondicionado con una cámara Hitachi KP-D 50 Color digital; algunas especies fueron observadas en Microscopio Electrónico de Barrido (MEB) para su análisis detallado: los especimenes fueron aislados con micropipetas y se enjuagaron (libres de sal y preservadores), se colocaron en cubreobjetos, se secaron 


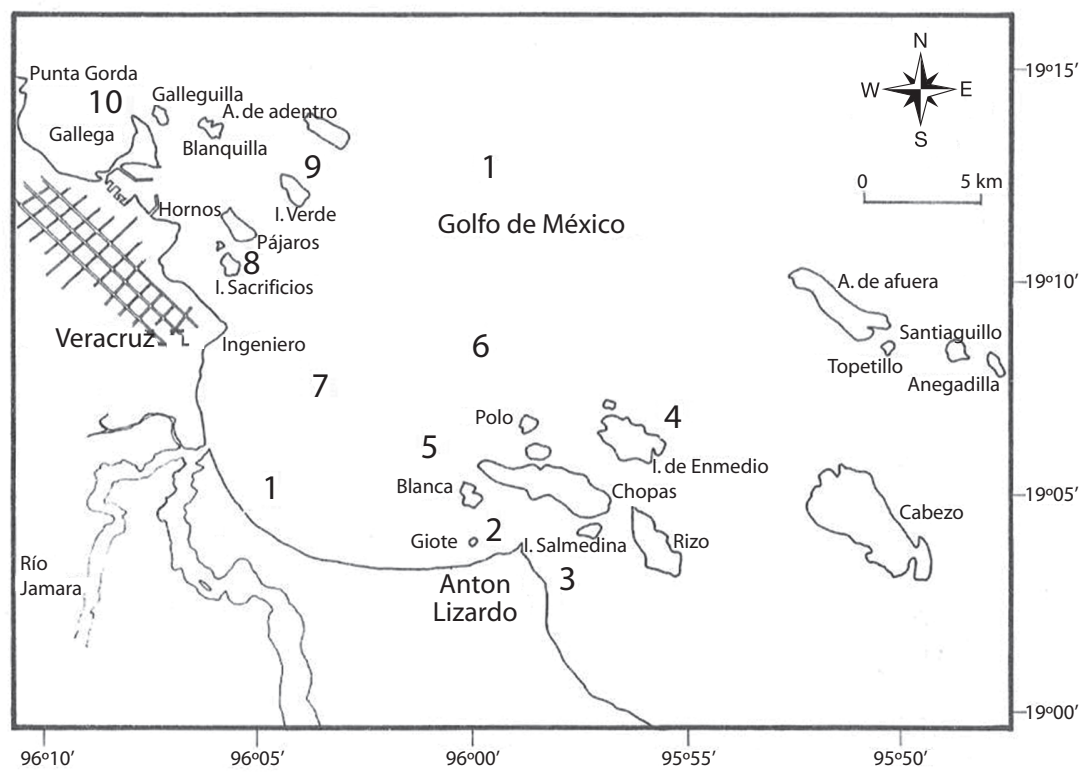

Fig. 1. Estaciones de muestreo en el Sistema Arrecifal Veracruzano.

Fig. 1. Sampling stations in the Sistema Arrecifal Veracruzano.

a temperatura ambiente y posteriormente se metalizaron con oro para su observación en el equipo JEOL JSM6360LV.

Para la identificación de las especies, se consideraron los caracteres taxonómicos y terminología propuestos por Dodge (1975), Sournia (1986), Steindinger \& Tangen (1996) y Tenenbaum (2006), además se utilizaron otros textos de consulta, como Osorio-Tafall (1942), Abé (1967), Wood (1968), Dodge (1975), Taylor (1976), Sournia (1986), Balech (1988), Licea et al. (1995), Steindinger \& Tangen (1996), Faust \& Gulledge (2002) y Tenenbaum
(2006). La clasificación taxonómica adoptada en esta investigación fue la de Fensome et al. (1993).

\section{RESULTADOS}

Se identificaron 9 especies de cuatro géneros, Dinophysis y Prorocentrum estuvieron representados por tres especies, dos de Phalacroma y una de Ornithocercus. A continuación se presentan claves para la identificación de órdenes, géneros y especies de dinoflagelados.

\section{Clave para determinar órdenes}

1. Dinoflagelados con la teca compuesta por dos valvas unidas lateralmente, con ausencia de cingulum y sulcus, con o sin espina en la parte anterior $\ldots \ldots \ldots \ldots \ldots \ldots \ldots \ldots \ldots \ldots \ldots \ldots \ldots \ldots \ldots \ldots \ldots \ldots \ldots \ldots \ldots$ Procentrales

1a. Dinoflagelados con la teca compuesta por dos valvas unidas lateralmente, con cingulum y sulcus $\ldots \ldots \ldots \ldots .2$

2. Dinoflagelados con aletas sulcales y cingulares radiadas o no $\ldots \ldots \ldots \ldots \ldots \ldots \ldots \ldots \ldots \ldots$ Dinophysiales 


\section{Clave para determinar géneros}

1. Células piriformes, cordiformes, sigmoides, compuesta por dos valvas, unidas lateralmente, con ausencia de cíngulo y sulcus, con una espina anterior.

Prorocentrum

1a. Células circulares, ovoides o irregulares con aletas cingulares radiadas o no. $\ldots \ldots \ldots \ldots \ldots \ldots \ldots \ldots$

2. Célula circular con la teca cubierta por poros, con dos aletas cingulares apicales próximas entre si y radiadas, con aleta sulcal izquierda radiada membranosa que se prolonga por debajo de la hipoteca . . . . . . . . . . Ornithocercus

2a. Célula ovoide o irregular, con aleta sulcal izquierda que no se prolonga por debajo de la hipoteca, y además con aleta

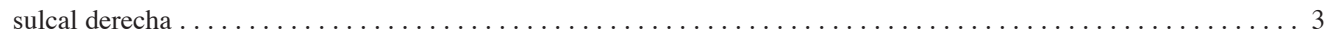

3. Célula con epiteca no prominente, teca en ocasiones con prolongaciones angulosas o a manera de espina o cuerno con

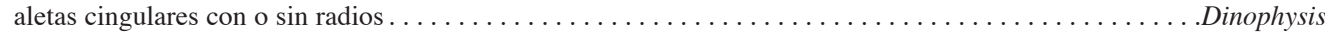

3a. Célula con epiteca prominente, teca en ocasiones con prolongaciones angulosas a manera de espina, con aletas singulares radiadas o no

Phalacroma

\section{Clave para determinar especies del orden Prorocentrales}

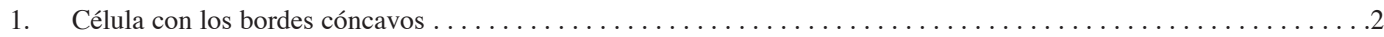

1a. Célula con uno de los bordes de la teca semi-recto y el otro borde cóncavo en la parte anterior y mas o menos recto en

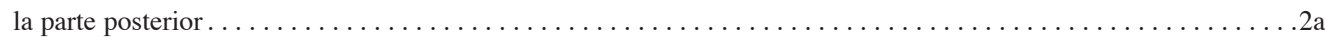

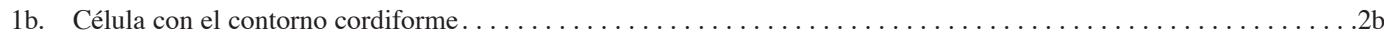

2. Célula con longitud de: $40-75 \mu \mathrm{m}$; ancho mayor de: $17.5-30 \mu \mathrm{m}$ y la longitud de la espina de: $7.5-15 \mu \mathrm{m}$; con poro oral

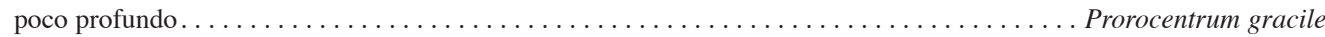

2a. Célula con longitud de: $67.5-72.5 \mu \mathrm{m}$; ancho mayor de: $20-25 \mu \mathrm{m}$ y la longitud de la espina de: $15-17.5 \mu \mathrm{m}$; con poro

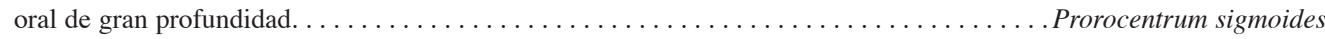

2b. Célula con longitud de: 32.5-67.5 $\mu \mathrm{m}$; ancho mayor de: $20-30 \mu \mathrm{m}$ y la longitud de la espina de: 5-10 $\mu \mathrm{m}$; con poro oral

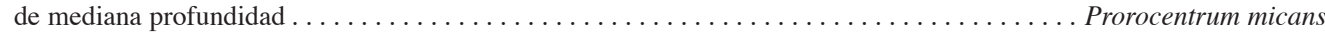

\section{Clave para determinar especies del orden Dinophysiales}

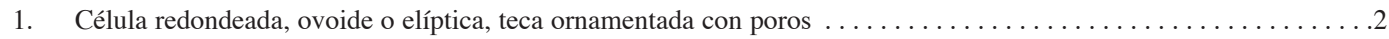

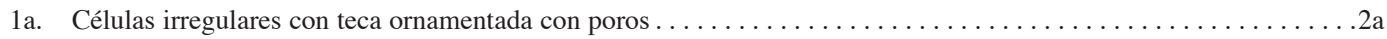

1b. Célula trapezoidal con aletas cingulares a la misma altura de la epiteca. $\ldots \ldots \ldots \ldots \ldots \ldots \ldots \ldots \ldots \ldots \ldots \ldots \ldots \ldots \ldots$

1c. Célula triangular con aletas cingulares por debajo de la epiteca prominente $\ldots \ldots \ldots \ldots \ldots \ldots \ldots \ldots \ldots \ldots$

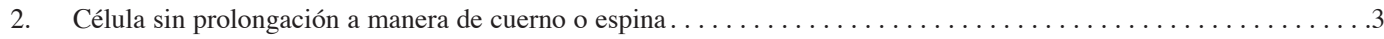

2a. Célula con prolongación como espina o cuerno por debajo de la hipoteca $\ldots \ldots \ldots \ldots \ldots \ldots \ldots \ldots \ldots \ldots \ldots \ldots \ldots \ldots \ldots \ldots$

3. La célula presenta aleta sulcal izquierda trilobulada por debajo de la hipoteca, además con tres radios sosteniendo el

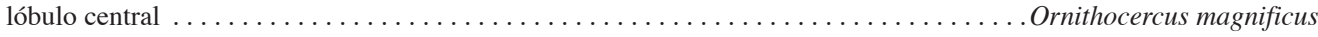

3a. Célula con la aleta sulcal izquierda recta, lateral, con tres $\operatorname{radios}(\mathrm{R} 1, \mathrm{R} 2, \mathrm{R} 3) \ldots \ldots \ldots \ldots$

4. La aleta sulcal izquierda con la misma distancia entre R1, R2 y R3 ................ Dinophysis caudata

4a. La aleta sulcal izquierda con diferente distancia entre los radios y además epiteca no prominente $\ldots \ldots \ldots \ldots . \ldots$

4b. La aleta sulcal izquierda con diferente distancia entre los radios y además epiteca prominente $\ldots \ldots \ldots \ldots \ldots$ a

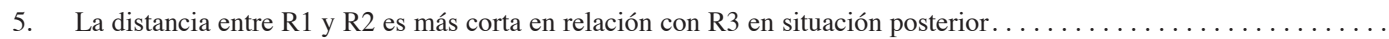

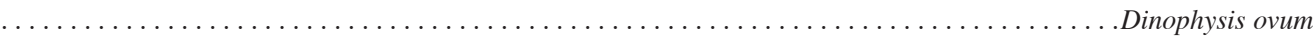

5a. La distancia entre R2 y R3 es más corta en situación posterior, que con R1 en situación anterior

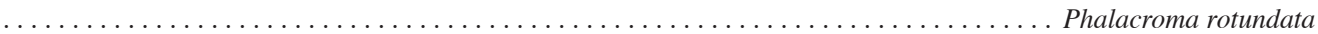

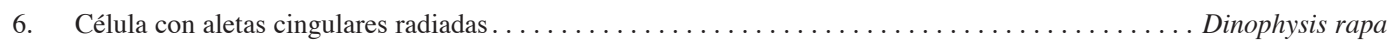

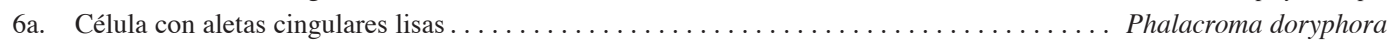




\section{Descripción de especies}

Se describen 9 especies identificadas, incluyendo las principales dimensiones: Longitud total (Lt), Longitud de la célula ( $\mathrm{Lc}$ ), Ancho total (At), Ancho de la célula (Ac), Espina apical (Ea), distancias entre R1 y R2 (R1-R2) y de R2 a R3 (R2-R3) (Figs. 2 y 3). Asimismo, se anotan datos de ocurrencia en el SAV, distribución general y en México y en los casos correspondientes, toxicidad.

División Dinoflagellata (Bütschli)

Fensome et al.

Clase Dinophyceae Pascher

Orden Prorocentrales Lemmermann

Familia Prorocentraceae Stein

Género Prorocentrum Ehrenberg

Prorocentrum gracile Schütt (Figs. 6 y 7)

Referencias: Schiller, 1933, p. 37, figs 39 a, b; Dodge 1975, p. 114, fig. 3C; Taylor, 1976, p. 22, pl. 1 fig. 2; Balech, 1988, p. 32, lam. 4, fig. 2; Steidinger \&Tangen, 1996, p. 423, pl. 8; Cohen-Fernández et al., 2006, p. 333, figs. 5, 6 y $20-25$.
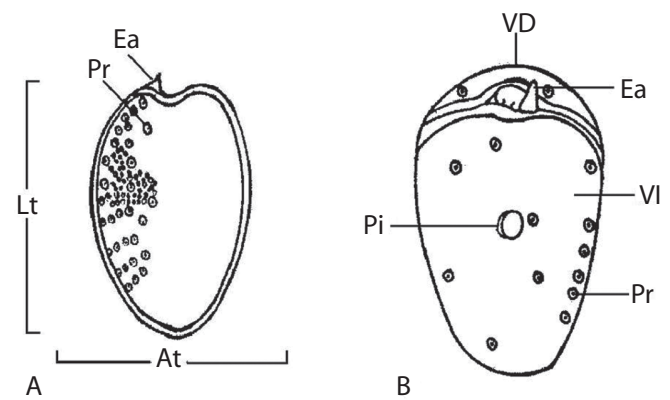

Fig. 2. Organización general de los Prorocentrales. a) Vista valvar derecha, Ea: espina apical, Pr: poros, Lt: longitud total, Dt: diámetro total; b) Vista valvar izquierda, VD: valva derecha, Ea: Espina apical, VI: valva izquierda, Pr: poros, Pi: pirenoide. Modificado de Steindinger \& Tangen (1996).

Fig 2. Prorocentrales general organization. a) Right valve view Ea: apical spine, Pr: pores, Lt: total length, Dt: total diameter; b) Left valve view, VD: right valve, Ea: apical spine, VI: left valve, Pr: pores, Pi: pyrenoid. Modified from Steindinger \& Tangen (1996).
Células solitarias, de talla mediana. La teca es redondeada en la parte anterior, sus bordes son cóncavos, suele terminar en punta, en algunas célula la punta se puede encontrar truncada o poco redondeada. El poro oral se observa poco profundo, la espina se observa desarrollada pero no muy larga. La sutura longitudinal en donde se unen las dos valvas no presenta ningún tipo de ornamentación, la teca está cubierta por una serie de poros distribuidos uniformemente, algunos de los poros se observan menos profundos que otros. Dimensiones: Lc: $40-75 \mu \mathrm{m},(60.8 \mu \mathrm{m})$; Ac: $17.5-30 \mu \mathrm{m}$, $(22.4 \mu \mathrm{m})$; Ea $7.5-15 \mu \mathrm{m},(10.8 \mu \mathrm{m})$.

Ocurrencia en el área de estudio: estaciones 1-3, 5, 7 y 10, octubre, 2006, 1-10 excepto 4 noviembre, 2006, 8 diciembre, 2006 y 4 enero, 2007.

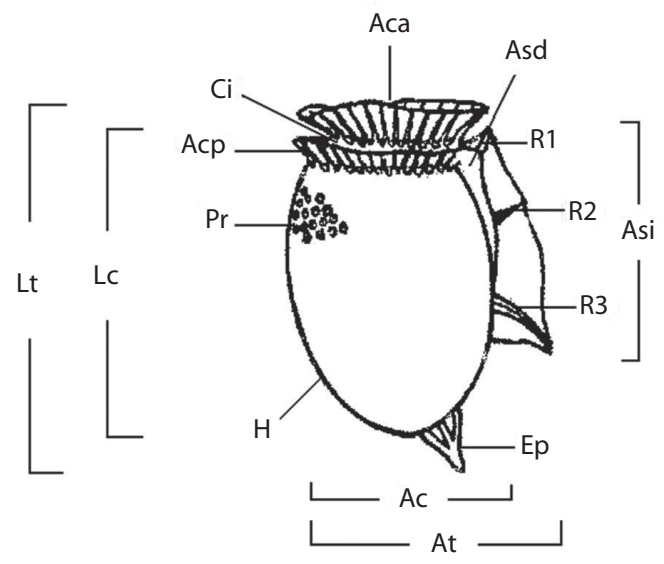

Fig. 3. Organización general de los Dinophysiales (Dinophysis) en vista lateral derecha; Lt: longitud total, Lc: longitud de la célula, Dt: diámetro total, Dc: diámetro de la célula, Aca: aleta cingular anterior, Ci: cíngulo, Acp: aleta cingular posterior, Pr: poros, H: hipoteca, Ep: espina, Asi: aleta sulcal izquierda, R1, R2 y R3: radios de la sulcal izquierda, Asd: aleta sulcal derecha. Modificado de Steindinger \& Tangen (1996).

Fig. 3. General organization of the Dinophysiales (Dinophysis) in right lateral view; Lt: total length, Lc: length of the cell, Dt: total diameter, Dc: diameter of the cell, Aca: anterior girdle list, Ci: cingulum, Acp: posterior girdle list, Pr: pores, H: hypotheca, Ep: spine, Asi: left sulcal list, R1, R2 y R3: ribs of the left sulcal list, Asd: right sulcal list. Modified from Steindinger \& Tangen (1996). 

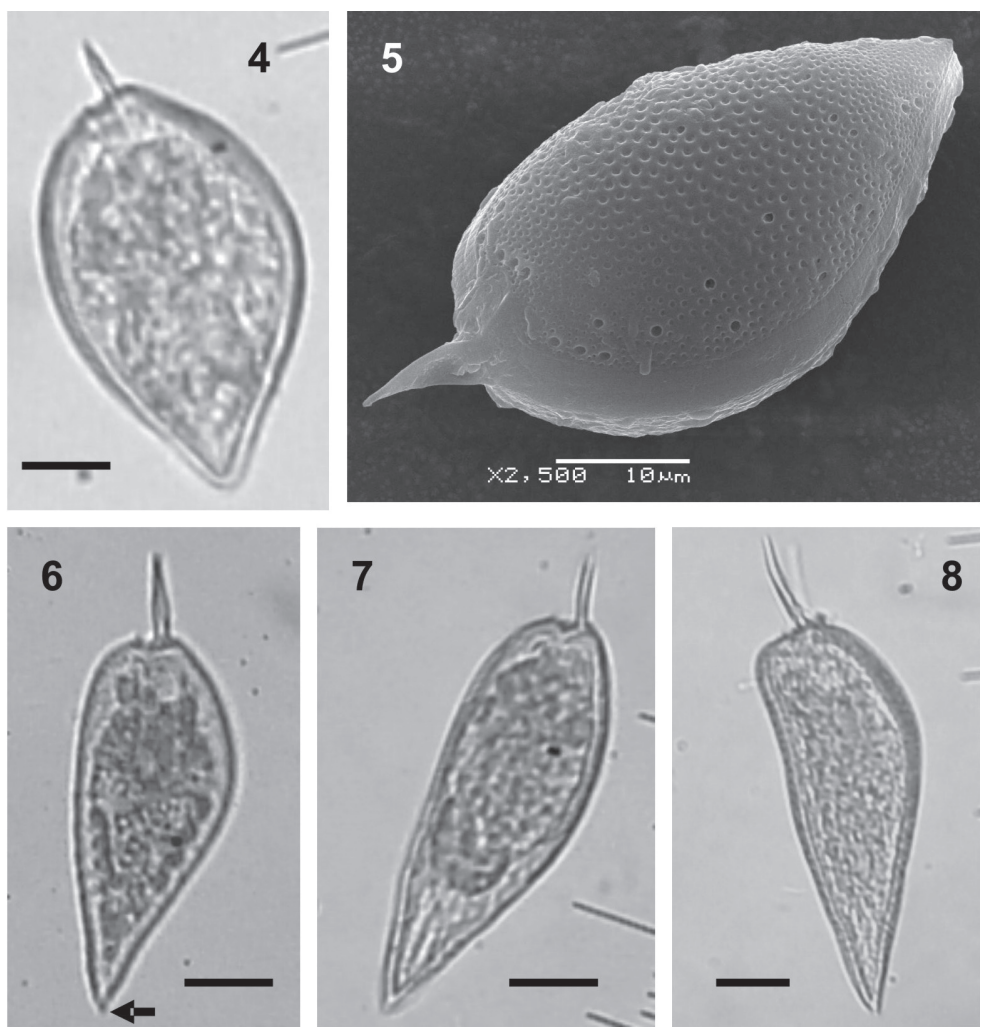

Figs. 4-8. Especies del género Prorocentrum. Figs. 4 y 5. Prorocentrum micans, ML y MEB respectivamente. Figs. 6 y 7. Prorocentrum gracile, ML. Fig. 8. Prorocentrum sigmoides, ML. Escala: $10 \mu \mathrm{m}$.

Figs. 4-8. Species of the genus Prorocentrum. Figs. 4 and 5. Prorocentrum micans, LM and SEM respectively. Figs. 6 and 7. Prorocentrum gracile, LM. Fig. 8. Prorocentrum sigmoides, LM. Scale: $10 \mu \mathrm{m}$.

Distribución general: Atlántico, Pacifico y Mediterráneo (Dodge 1975), preferencia por aguas cálidas (Hernández-Becerril et al. 2003).

Distribución en México: Ampliamente distribuida en el Pacifico mexicano (Hernández-Becerril et. al.2000).

\section{Prorocentrum micans Ehrenberg (Figs. 4 y 5)}

Referencias: Dodge 1975, p. 112, fig. 3A; Taylor 1976, p. 23, pl. 1 fig. 1; Balech 1988, p. 32, lam. 4, fig. 1; Steidinger \& Tangen 1996, p. 424, pl. 8; Cohen-Fernández et al. 2006, p.
332, figs. 3, 4 y 15-18; Fukuyo et al. 1990: p. 29, figs. $28 \mathrm{~A}-\mathrm{F}$.

Células solitarias y de talla mediana. La forma de la teca de esta especie es muy variable, ya que pueden ir desde células piriformes hasta células en forma de corazón, los bordes de la célula son cóncavos. En muchos casos no existe gran diferencia entre al largo y ancho de la célula, cabe destacar que la parte posterior de esta termina en una pequeña punta no prolongada. El poro oral es menos profundo que en $P$. sigmoides, posee una espina mediana y delgada. La teca está cubierta por poros de diferentes dimensiones y profundidades, acomodados de 
manera especial, si se observa al microscopio electrónico de barrido se observan poros alargados arreglados en forma de $\mathrm{V}$ en la parte posterior de la teca, dos líneas de poros un poco más arriba en forma de V invertida. Dimensiones: Lc: $32.5-67.5 \mu \mathrm{m}(41.9 \mu \mathrm{m})$; Ac: $20-30 \mu \mathrm{m}$ (25.1 $\mu \mathrm{m})$; Ea: 5-10 $\mu \mathrm{m}(8.1 \mu \mathrm{m})$.

Ocurrencia en el SAV: estaciones 1, 2, 5, 8 octubre, 2006; 1, 6, 8, 10 noviembre, 2006; 9 diciembre, 2006; 1, 3, 4, 6-10 enero, 2007.

Distribución general: Planctónica, nerítica y estuarina (Steindinger \& Tangen 1996). Es común encontrarla en los mares de todo el mundo (Dodge 1975).

Distribución en México: Ampliamente distribuida en el Pacífico Mexicano (Hernández-Becerril et. al. 2000), común en aguas costeras del Pacífico mexicano y el Golfo de México (Cortés- Altamirano \& HernándezBecerril, 1998).

\section{Prorocentrum sigmoides Böhm (Fig. 8)}

Referencias: Osorio-Tafall 1942, p. 438; Dodge 1975, p. 113, fig. 3C; Cohen-Fernández et al. 2006, p. 333, figs. 7, 8, 26-30.

Células solitarias, de medianas a grandes. Células con la teca muy alargada, casi siempre termina en punta muy prolongada, uno de los bordes de la teca es recto mientras que el otro es un tanto cóncavo en la parte anterior y recto en la parte posterior. La parte anterior de la teca es más o menos ancha y poco redondeada, ahí se puede observar que el poro oral es profundo y la espina es muy larga y delgada, cuando se analiza al microscopio óptico es común observar dentro de la célula materia orgánica. La superficie de la teca esta cubierta por poros de igual profundidad y tamaño. Dimensiones: Lc: $67.5-72.5 \mu \mathrm{m}(69.7 \mu \mathrm{m})$; Ac: $20-25 \mu \mathrm{m}$ $(23.05 \mu \mathrm{m})$ y Ea: $15-17.5 \mu \mathrm{m}(16.1 \mu \mathrm{m})$.

Ocurrencia en el área de estudio: estaciones 1 , octubre y noviembre, 2006, 9 diciembre, 2006.
Distribución general: Preferencia por aguas cálidas (Hernández-Becerril et al. 2003)

Distribución en México: Costas del Pacifico mexicano (Cohen-Fernández et al. 2006).

Orden Dinophysiales Kofoid

Familia Dinophyceae Stein

Género Dinophysis Eherenberg

Dinophysis caudata Saville-Kent (Figs. 9, 10)

Referencias: Schiller 1933, p. 152, fig. 145 a-u; Abé 1967, p. 56, fig. 14 a-d; Taylor 1976, p. 34, pl. 6, fig. 59; Balech 1988, p. 45, lam. 8, fig. 2-3; Fukuyo et al. 1990, p. 37, fig. 36; Steidinger \& Tangen, 1996, p. 431, pl. 12; Tenenbaum 2006, p. 117.

Las células de esta especie poseen una teca de tamaño considerable, su cuerpo es amplio, en la parte posterior (hipoteca) tienen una prolongación que las distingue de las de más especies de este género; por arriba de la epiteca tienen un par de aletas cingulares multiradiadas. Tienen dos aletas sulcales, la sulcal izquierda va desde la parte posterior de la epiteca hasta antes de la prolongación de la hipoteca esta sostenida por tres radios (R1, R2 y R3), la sulcal derecha es más pequeña y generalmente llega hasta R2. Se encuentra frecuentemente agrupada en pares. Dimensiones: Lt: $70-107.5 \mu \mathrm{m}(89.5 \mu \mathrm{m}), \mathrm{Lc}: 60-95 \mu \mathrm{m}$ $(78.5 \mu \mathrm{m})$, At: $35-80 \mu \mathrm{m}(58.5 \mu \mathrm{m})$, Ac: $27.5-$ $67.5 \mu \mathrm{m}(44.1 \mu \mathrm{m}), \mathrm{R} 1-\mathrm{R} 2: 7.5-15 \mu \mathrm{m}(10.5 \mu \mathrm{m})$, R2-R3: $10-15 \mu \mathrm{m}(13.1 \mu \mathrm{m})$.

Ocurrencia en el SAV: estaciones 1-3 y 10 , octubre $2006,1,2,5,6,9$ y 10 noviembre 2006, 2, 8-10 diciembre 2006, 1-10 excepto 2 y 6 , enero 2007 .

Distribución general: Nerítica y estuarina en aguas templadas a tropicales; raramente encontrada en aguas frías, posible intruso en masas de agua templada (Steindinger \& Tangen 1996).

Distribución en México: Común en aguas costeras del Pacífico mexicano y el Golfo 

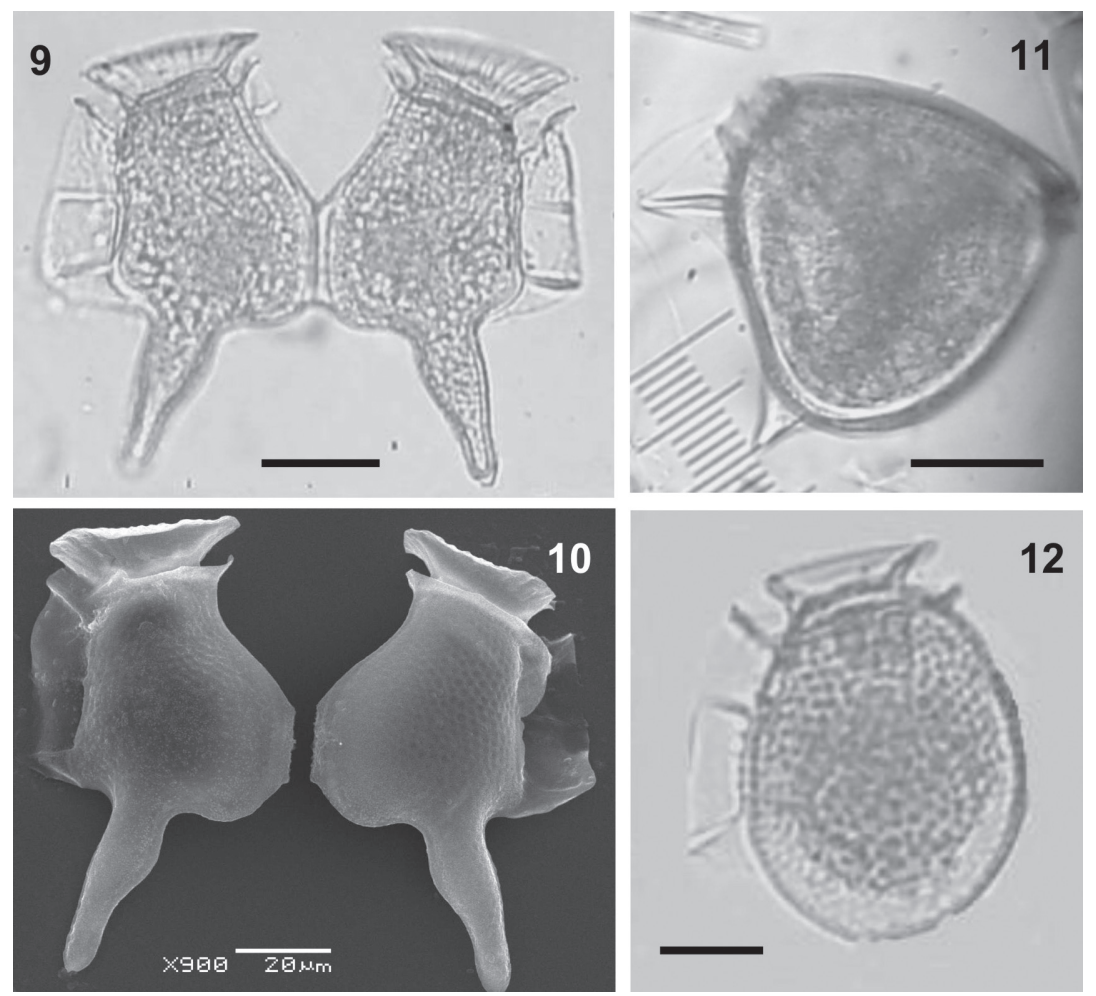

Figs. 9-12. Especies de los géneros Dinophysis y Phalacroma. Figs. 9, 10. Dinophysis caudata, ML y MEB respectivamente. Fig. 11. Phalacroma doryphora, vista lateral izquierda, ML. Fig. 12. Dinophysis ovum, vista lateral izquierda, ML. Escala: $20 \mu \mathrm{m}$.

Figs. 9-12. Species of the genera Dinophysis and Phalacroma. Figs. 9, 10. Dinophysis caudata, LM and SEM respectivly. Fig. 11. Phalacroma doryphora, left lateral view, LM. Fig. 12. Dinophysis ovum, left lateral view, LM. Scale: $2 \mu \mathrm{m}$.

de México (Cortés-Altamirano \& HernándezBecerril 1998).

Productora de acido okadaico, implicado con DSP (Fukuyo et al.1990).

Dinophysis ovum Schütt (Fig. 12)

Referencias: Schiller 1933, p. 116, fig. 109; Abé 1967, p. 50, figs. 10 a-p.

Célula de tamaño mediano, la teca tiene forma ovalada a elíptica además de estar cubierta por una serie de poros regulares. Poseen dos aletas cingulares, la aleta cingular anterior por arriba de la epiteca, mientras que la cingular posterior se encuentra por debajo de la epiteca; ambas lisas. La aleta sulcal izquierda se encuentra bien definida y se observan los tres radios que la sostienen, R3 se ve ligeramente más larga que R1 y R2, esta ultima visiblemente mas cerca de R1 que de R3; la sulcal derecha no se observa ya que se encuentra en vista izquierda y sólo es posible observarla en vista derecha. Dimensiones: Lt: $47.5 \mu \mathrm{m}$, Lc: $45 \mu \mathrm{m}$, At: $42.5 \mu \mathrm{m}$, Ac: $35 \mu \mathrm{m}$, R1-R2: $7.5 \mu \mathrm{m}, \mathrm{R} 2-\mathrm{R} 3: 12.5 \mu \mathrm{m}$.

Ocurrencia en el SAV: estación 2, diciembre 2006.

Distribución general: Adriático y Antártico, Golfo de Finlandia, Océano Atlántico, Mar Mediterráneo y aguas costeras del noroeste del Pacífico. (Abé 1967) 
Distribución en México: Pacifico mexicano específicamente en localidades como Jalisco, Colima y Michoacán (Hernández-Becerril et. al. 2008).

Especie relacionada con producción de ácido okadaico (toxina responsable de DSP) (Swanson et al. 2010).

\section{Dinophysis rapa (Stein) Abé (Fig. 13)}

Referencias: Abé 1967, p. 66, fig. 19; Taylor 1976, p. 40, pl. 5, figs. 48 a, b, pl. 41, fig. 488; Balech 1988, p. 44, lam. 8, figs. 6-8.

Célula con la teca de forma irregular (trapezoidal) y cubierta de poros regulares, aletas cingulares muy cerca entre si, la aleta cingular anterior multiradiada y ubicada a la misma altura del borde superior de la epiteca; en vista superior se observa la cingular anterior a manera de corona. Presenta aleta sulcal izquierda sostenida por tres radios (R1, R2 y R3). La parte inferior de la aleta sulcal izquierda se observa más amplia que la parte superior de la misma; lo que hace suponer que R3 es mucho más largo que los otros dos radios; la hipoteca termina en forma de cuerno. Dimensiones: Lc: $77.5-87.5 \mu \mathrm{m}(82.5 \mu \mathrm{m})$, At: $72.5-77.5 \mu \mathrm{m}$ (75 $\mu \mathrm{m})$, Ac: $65-67.5 \mu \mathrm{m}(66.2 \mu \mathrm{m}), \mathrm{R} 1-\mathrm{R} 2: 7.5-$ $10 \mu \mathrm{m}(8.7 \mu \mathrm{m}), \mathrm{R} 2-\mathrm{R} 3: 10 \mu \mathrm{m}$.

Ocurrencia en el SAV: estaciones 9 y 10 , octubre 2006.

Distribución general: Atlántico norte, sur y Océano Indico (Licea et al. 1995). Especie de aguas cálidas, quizás algo umbrófila (Balech 1988).

Distribución en México: Jalisco, Colima y Michoacán (Hernández-Becerril et al. 2008).

Se ha encontrado como productora de ácido okadaico (Zingone 2001).

Género Ornithocercus Stein

Ornithocercus magnificus Stein (Figs. 17, 18)

Referencias: Schiller 1933, p. 198, figs 190 a, b; Balech 1988, p. 61, lam. 14, figs. 7, 8; Steidinger \& Tangen 1996, p. 436, pl. 13.
Las células de éste género constan de una teca provista con Pr, por arriba de la epiteca podemos encontrar dos aletas cingulares que dan la apariencia de una corona, Ca y $\mathrm{Cp}$, ambas bien desarrolladas y multiradiadas. La aleta sulcal a diferencia de otros géneros se prolonga hasta por debajo de la hipoteca y una de las características de esta especie es que esta aleta es trilobulada, en el lóbulo central podemos encontrar tres radios (R1, R2 y R3) los cuáles sostienen este; así mismo, es muy común encontrar cianobacterias simbiontes llamadas phaeosomas entre las aletas cingulares. Dimensiones: Lt: 95-105 $\mu \mathrm{m}(101.7 \mu \mathrm{m})$, Lc: $37.5-45 \mu \mathrm{m}(42.1 \mu \mathrm{m})$, At: $62.5-82.5 \mu \mathrm{m}$ (75 $\mu \mathrm{m})$, Ac: $40-45 \mu \mathrm{m}(42.5 \mu \mathrm{m})$.

Ocurrencia en el SAV: estaciones 8-10, octubre, 2006, 2 y 5, noviembre, 2006, 3 у 9, diciembre, 2006.

Distribución general: Cosmopolita de aguas tropicales y Mar Mediterráneo (Licea et. al. 1995).

Distribución en México: Pacífico Central y Golfo de Tehuantepec (Hernández-Rosas et. al. 2007).

Género Phalacroma Stein

Phalacroma doryphora Stein (Fig. 11)

Sinónimo: Dinophysis doryphora (Stein) Abé.

Referencias: Norris \& Berner 1970, p. 161, figs. 23-33; Taylor 1976, p. 35, pl. 4, figs. 41, 42; Balech 1988, p. 55, lam. 13, figs. 11-13; Jensen \& Daugbjerg 2009, p. 1 139, fig. 3-i.

Célula de mediana a grande, la teca es de forma triangular y con poros dispersos, las aletas cingulares se encuentran muy juntas entre si y a la altura de la epiteca, la aleta sulcal izquierda abarca poco menos de la mitad de la teca, posee tres radios, es mas ancha a la altura de R3 lo que hace suponer que R3 es mas larga que R1 y R2. La principal característica de $P$. doryphora aparte de la forma de la teca es que 
por debajo de la hipoteca posee un pequeño apéndice triangular. Dimensiones: Lc: $67.5 \mu \mathrm{m}$, At: $85 \mu \mathrm{m}$, Ac: $62.5 \mu \mathrm{m}, \mathrm{R} 2-\mathrm{R} 3: 7.5 \mu \mathrm{m}$.

Ocurrencia en el SAV: estación 8, diciembre 2006.

Distribución general: Termófila, tropical (Licea et. al. 1995).

Distribución en México: Desde Bahía Magdalena, B.C.S. hasta Chiapas (HernándezBecerril et al. 2008).

\section{Phalacroma rotundata}

(Claparède et Lachmann) Kofoid et Michener (Figs. 14-16)

Sinónimo: Dinophysis rotundata Claparède et Lachmann

Referencias: Schiller 1933, p. 67, figs. 60 a-d; Abé 1967, p. 57, fig. 15 a-h; Balech 1988, p. 46, lam. 9, figs 1-4; Hallegraeff et al. 1995: 307, 308, fig. 15.30, Jensen \& Daugbjerg 2009, p. 1142.

Célula de forma redondeada o hasta ovalada, la superficie de la teca cubierta por poros; la epiteca es mas redondeada que la hipoteca y algo sobresaliente, tanto la aleta cingular anterior como la posterior son angostas y lisas. $\mathrm{La}$ aleta sulcal izquierda abarca un poco mas de la mitad de la teca y se encuentra sostenida por tres radios, $\mathrm{R} 2$ mas cerca de $\mathrm{R} 3$ que de $\mathrm{R} 1$. La sulcal derecha es casi del mismo ancho que la sulcal izquierda y se observa extendida hasta R3. Dimensiones: Lc: $35-52 \mu \mathrm{m}(41.7 \mu \mathrm{m})$, At: $37.5-52.5 \mu \mathrm{m}(43.5 \mu \mathrm{m})$, Ac: $30-45 \mu \mathrm{m}$ $(35.7 \mu \mathrm{m}), \quad \mathrm{R} 1-\mathrm{R} 2: \quad 7.5-12.5 \mu \mathrm{m} \quad(9.16 \mu \mathrm{m})$, R2-R3: 5-7.5 $\mu \mathrm{m}(5.31 \mu \mathrm{m})$.

Ocurrencia en el SAV: estaciones 10, octubre 2006, 2, noviembre 2006, 1, 2, 8-10 diciembre 2006, 4, enero 2007.

Distribución general: Atlántico oriental, en aguas neríticas y mares europeos (Licea et al. 1995).
Distribución en México: Desde Jalisco hasta Chiapas (Hernández-Becerril et al. 2008).

Especie toxica relacionada con Envenenamiento Diarreico por consumo de Mariscos, productora de la toxina Dinophysistoxina-1 (DTX1) (Faust \& Gulledge 2002).

\section{DISCUSIÓN}

El número de especies encontradas en este trabajo puede ser considerado bajo, con solo 9 especies identificadas, sobre todo considerando las 252 especies de dinoflagelados planctónicos registrados por Licea et al. (2004) en varios cruceros del sur del Golfo de México, o las 46 especies de dinoflagelados planctónicos del género Protoperidinium encontradas en este mismo sistema (SAV) por Okolodkov (2008). Esto puede deberse en parte al período corto estudiado o las condiciones ambientales particulares del mismo período. Análisis reciente de material fitoplanctónico de red proveniente de cruceros en el sureste del Golfo de México, revelan mayor diversidad de Dinophysiales en aguas oceánicas que costeras (Parra-Toriz, en prep.).

Algunas especies que fueron comunes en el trabajo de Licea et al. (2004), se encontraron igualmente comunes en el presente trabajo, como Dinophysis caudata, Ornithocercus magnificus, Prorocentrum gracile y P. micans; todas, a excepción de $O$. magnificus, estuvieron presentes durante los cuatro meses muestreados en este estudio. Diciembre fue el mes con mayor ocurrencia de especies con ocho especies de las nueve identificadas. Es importante mencionar que la especie Dinophysis ovum, encontrada en este trabajo, no fue registrada por Licea et al. (2004), aunque si aparecieron otras varias especies del género Dinophysis. Esta especie se encontró en el norte del Golfo de México (costas de Texas, E.U.) formando proliferaciones, en enero y febrero de 2008 (Swanson et al. 2010).

Existen antecedentes que muestran importantes proliferaciones y eventos tóxicos causados por el dinoflagelado atecado Karenia brevis en la zona de estudio (SAV), a finales 

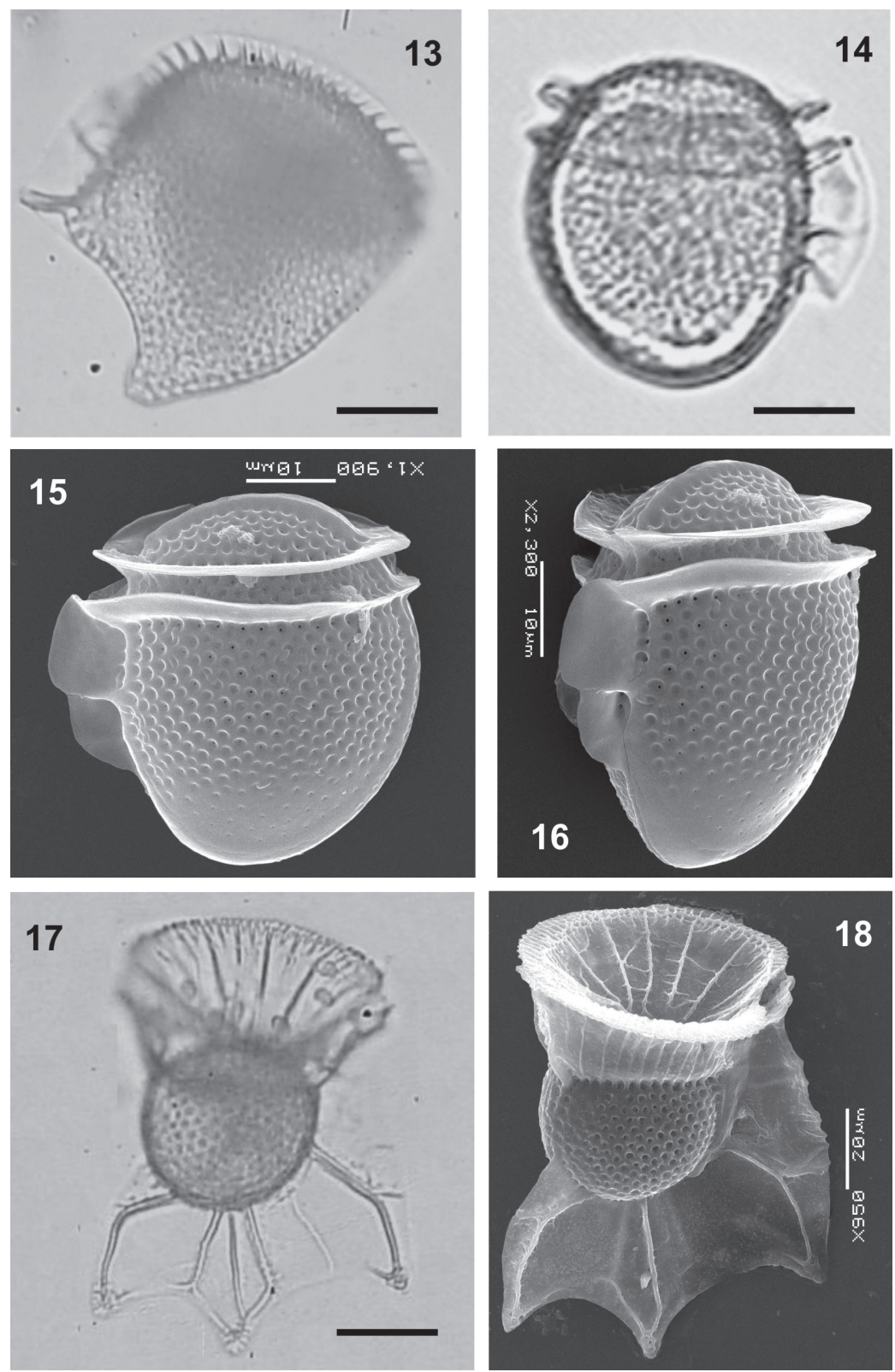

Figs. 13-18. Especies de los géneros Dinophysis, Phalacroma y Ornithocercus. Fig. 13. Dinophysis rapa, ML. Figs. 14-16. Phalacroma rotundata, vista lateral derecha (ML), y vista lateral izquierda (MEB). Figs. 17, 18 Ornithocercus magnificus, vista lateral derecha, ML y MEB respectivamente. Escala: 10 $\mu \mathrm{m}$ (Figs. 14-16), 20 $\mu \mathrm{m}$ (Figs. 13, 17, 18).

Figs. 13-18. Species of the genera Dinophysis, Phalacroma and Ornithocercus. Fig. 13. Dinophysis rapa, LM. Figs. 14-16. Phalacroma rotundata, right lateral view (LM) and left lateral view (SEM). Figs. 17, 18. Ornithocercus magnificus, right lateral view, LM y SEM respectively. Scale: $10 \mu \mathrm{m}$ (Figs. 14-16), $20 \mu \mathrm{m}$ (Figs. 13, 17, 18). 
de 2000 y principio de 2001, ocasionando muerte de fauna marina y daños a las pesquerías (Tester et al. 2004). No existe información publicada recientemente sobre otros eventos en la zona de estudio o áreas aledañas, especialmente relacionada con las especies tratadas en este trabajo.

\section{AGRADECIMIENTOS}

Se agradece a Yolanda Hornelas (USMEB, ICMyL, UNAM) por su colaboración en el empleo del microscopio electrónico de barrido. Dos árbitros anónimos contribuyeron con sus críticas para mejorar el primer manuscrito sometido. La primera autora (D. P.-T.) recibió beca por parte de un proyecto de investigación apoyado por PAPIIT, DGAPA, UNAM (IN226309).

\section{RESUMEN}

Los dinoflagelados son considerados como el grupo más abundante y diverso de microalgas del fitoplancton después de las diatomeas. Esta investigación da a conocer la composición específica de dinoflagelados planctónicos de los ordenes Prorocentrales y Dinophysiales, recolectados mediante arrastres superficiales con red $(30 \mu \mathrm{m}$ de luz de malla) en el Sistema Arrecifal Veracruzano (SAV), de las costas de Veracruz, México, en el período octubre 2006 a enero2007. Para el análisis del material se utilizaron microscopios de luz y electrónico de barrido. Se identificaron 9 especies de cuatro géneros: Dinophysis estuvo representado por 3 especies, al igual que Prorocentrum, dos de Phalacroma y una de Ornithocercus. Se presentan claves para identificación de los órdenes, géneros y especies de dinoflagelados, asimismo se describe e ilustra cada especie, incluyendo datos morfométricos y se menciona la distribución de las mismas. Dinophysis caudata, D. rapa, D. ovum y Phalacroma rotundata se han documentado como especies potencialmente productoras de toxinas. La riqueza de especies en el SAV se considera muy baja, especialmente considerando mayores riquezas en otras zonas del Golfo de México, como el sur del Golfo de México, donde se han listado 53 especies del orden Dinophysiales y 14 especies de Prorocentrales.

Palabras clave: dinoflagelados, fitoplancton, morfología, sistema arrecifal veracruzano, taxonomía.

\section{REFERENCIAS}

Abé, T.H. 1967. Armoured dinoflagellata. II. B. Dinophysis and its allied genera. Publ. Seto Mar. Biol. Lab. 15: 37-78.

Balech, E. 1988. Los Dinoflagelados del Atlántico Sudoccidental. Ministerio de Agricultura, Pesca y Alimentación, Madrid, España.

Cohen-Fernández, E., M.E. Meave del Castillo, I.H. Salgado Ugarte \& F.F. Pedroche. 2006. Contribution of external morphology in solving a species complex: The case of Prorocentrum micans, Prorocentrum gracile and Prorocentrum sigmoides (Dinoflagellata) from the Mexican Pacific coast. Phycol. Res. 54: 330-340.

Cortés-Altamirano, R. \& D.U. Hernández-Becerril. 1998. Especies responsables y probables de mareas rojas en México, p. 43-79. In R. Cortés-Altamirano (ed.). Las Mareas Rojas. AGT, México.

Dodge, J.D. \& B.T. Bibby. 1973. The Prorocentrales (Dinophyceae). I. A comparative account of the fine structure of the genera Prorocentrum and Exuviaella. Bot. J. Linn. Soc. 67: 175-187.

Dodge, J.D. 1975. The Prorocentrales (Dinophyceae). II. Revision of the taxonomy within the genus Prorocentrum. Bot. J. Linn. Soc. 71: 103-125.

Faust, M.A. \& R.A. Gulledge. 2002. Identifying Harmful Marine Dinoflagellates. Smithsonian, Washington D.C., EEUU.

Fensome, R.A., F.J.R. Taylor, G. Norris, W.A.S. Sarjeant, D.I. Wharton \& G.L. Williams. 1993. A classification of living and fossil dinoflagellates. Micropaleontology, Special Publication Number 7. Sheridan, Hanover, Pensilvania, EEUU.

Fukuyo, Y., H. Takano, M. Chihara \& K. Matsuoka. 1990. Dinophyceae. In H.G. Barber \& E.Y. Haworth (eds.). Red Tide Organisms in Japan. An Illustrated Taxonomic Guide. Uchida Rukakuho, Tokyo, Japan.

Gómez, F. 2005. A list of free-living dinoflagellate species in the world's oceans. Acta Bot. Croatica 64: 129-212.

Gutiérrez, D., C. García-Sáenz, M. Lara \& C. Padilla. 1993. Comparación de Arrecifes Coralinos: Veracruz y Quintana Roo, p. 787-806. In S.I. Salazar-Vallejo \& N.E. González (eds.). Biodiversidad Marina y Costera de México. CONABIO y CIQRO, México, D.F., México. 
Hallegraeff, G.M., D.M. Anderson \& A.D. Cembella. 1995. Taxonomy of Harmful Dinoflagellates. In UNESCO (ed.). IOC Manuals and Guide No. 33.

Hernández-Becerril, D.U. 2003. Diversidad del fitoplancton marino de México. Un acercamiento actual, p. 1-17. In M.T. Barreiro-Güemes, M.E. Meave del Casillo, M. Signoret-Poillon \& M.G. Figueroa-Torres (eds.). Planctología Mexicana. Sociedad Mexicana de Planctología, México D. F., México.

Hernández-Becerril, D.U., R. Alonso-Rodríguez, C. Álvarez-Góngora, S.A. Barón-Campis, G. Ceballos-Corona, J. Herrera-Silveira, M.E. Meave Del Castillo, N. Juárez-Ruíz, F. Merino-Virgilio, A. Morales-Blake, J.L. Ochoa, E. Orellana-Cepeda, C. Ramírez-Camarena \& R. Rodríguez-Salvador. 2007. Toxic and harmful marine phytoplankton and microalgae (HABs) in Mexican Coasts. J. Environ. Sci. Health Environ. Sci. Eng. 42: 1349-1363.

Hernández-Becerril, D.U., J.G.A. Ceballos-Corona, K. Esqueda-Lara, M.A. Tovar-Salazar, D. León-Álvarez. 2008. Marine planktonic dinoflagellates of the order Dinophysiales (Dinophyta) from coasts of the tropical Mexican Pacific, including two new species of the genus Amphisolenia. J. Mar. Biol. Assoc. U.K. 8: $1-15$.

Hernández-Becerril, D.U., R. Cortés-Altamirano \& R. Alonso. 2000. The dinoflagellate genus Prorocentrum along the coast of the Mexican Pacific. Hydrobiologia 418: 111-121.

Hernández-Becerril, D.U., M.E. Meave del Castillo \& C. Flores-Granados. 2003. Dinoflagelados del orden Dinophysiales en las costas mexicanas, p. 19-42. In M.T. Barreiro-Güemes, M.E. Meave del Casillo, M. Signoret-Poillon \& M.G. Figueroa-Torres (eds.). Planctología Mexicana. Sociedad Mexicana de Planctología, México, D.F., México.

Hernández-Rosas, A., M.E. Meave del Castillo, M.E. Zamudio-Resendiz \& M. Castillo Rivera. 2007. Morfometría y distribución de especies del género Ornithocercus (Dinophysiales: Dinophyta) del Pacífico Mexicano. Hidrobiológica 17: 257-272.

Licea, S., J.L. Moreno, H. Santoyo \& G. Figueroa. 1995. Dinoflageladas del Golfo de California. Universidad Autónoma de Baja California Sur, México D.F., México.

Licea, S., M.E. Zamudio, R. Luna \& J. Soto. 2004. Free-living dinoflagellates in the southern Gulf of Mexico: Report of data (1979-2002). Phycol. Res. 52: 419-428.
Jensen, M.H. \& N. Daugbjerg. 2009. Molecular phylogeny of selected species of the Order Dinophysiales (Dinophyceae) - testing the hypothesis of a Dinophysioid radiation. J. Phycol. 45: 1136-1152.

Norris, D.R. \& L.D. Berner. 1970. Thecal morphology of select species of Dinophysis (Dinoflagellata) from the Gulf of Mexico. Contr. Mar. Sci. 15: 145-192.

Okolodkov, Y.B. 2008. Protoperidinium Bergh (Dinophyceae) of the Nacional Park Sistema Arrecifal Veracruzano, Gulf of Mexico, with a key for identification. Acta Bot. Mex. 84: 93-149.

Okolodkov, Y.B., G. Campos-Bautista, I. Gárate-Lizárraga, J.A.G. González-González, M. Hoppenrath \& V. Arenas. 2007. Seasonal changes of benthic and epiphytic dinoflagellates in the Veracruz reef zone, Gulf of México. Aquat. Microb. Ecol. 47: 223-237.

Osorio-Tafall, B.F. 1942. Notas sobre algunos dinoflagelados planctónicos marinos de México con descripción de nuevas especies. An. Esc. Nac. Cienc. Biol. 2: 435-447.

Schiller, J. 1933. Dinoflagellatae (Peridineae), p. 617. In L. Rabenhorst (ed.). Kryptogamen Flora von Deutschland, Österreich und der Schweiz. Part I. Akademische s Gesellschaft. Geest y Portig K.-G. Leipzig.

Sournia, A. 1986. Atlas Du Phytoplancton Marin. Volume I: Introduction, Cyanophycées, Dictyophycées, Dinophycées et Raphidophycées. Editions du Centre National de la Recherche Scientifique, Paris, France.

Steidinger, K.A. \& K. Tangen. 1996. Dinoflagellates, p. 387-729. In C.R. Tomas (ed.). Identifying Marine Phytoplankton. Academic, San Diego, California, EEUU.

Swanson, K.M., L.J. Flewelling, M. Byrd, A. Nunez \& T.A. Villareal. 2010. The 2008 Texas Dinophysis ovum bloom: Distribution and toxicity. Harmful Algae 9: 190-199.

Taylor, F.J.R. 1976. Dinoflagellates from the International Indian Ocean expedition. A report on material collected by the R. V. Anton Bruun 1963-1964. Bibliotheca Botanica, Stuttgart, Alemania.

Taylor, F.J.R. 1987. General group characteristics, p. 1-23. In F.J.R. Taylor (ed.) The Biology of Dinoflagellates. Blackwell, Oxford, United Kingdom.

Taylor, F.J.R., M. Hoppenrath \& J.F. Saldarriaga. 2008. Dinoflagellate diversity and distribution. Biodivers. Conserv. 17: 407-418. 
Tenenbaum, D.R. 2006. Os Dinoflagelados, p. 111-161. In D.R. Tenenbaum (ed.). Dinoflagelados e Tintinídeos da Região Central da Zona Económica Exclusiva Brasileira: Guía de Identificacão. Museu Nacional Río de Janeiro, Brazil.

Tester, P.A., K. Wiles, S.M. Varnam, G. Velez-Vega, A.M. Dubois \& V. Arenas-Fuentes. 2004. Harmful algal blooms in the western Gulf of Mexico: Karenia brevis is messin' with Texas and Mexico, p. 41-43. In K.A. Steidinger, J.H. Landsberg, C.R. Tomas \& G.A. Vargo (eds.). Harmful Algae 2002. Florida Fish and Wildlife Conservation Commission and Florida Institute of Oceanography and Intergovermental Oceanographic Commission of UNESCO, St. Petersburg, EEUU.

Tunnell, W.J., E.A. Chavez \& K. Withers. 2007. Coral Reefs of the Southern Gulf of Mexico. Harte Research
Institute, Gulf of Mexico Series. Texas A \& M University, Corpus Christi, EEUU.

Vargas-Hernández, J.M., A. Hernández Gutiérrez \& L.F. Carrera Parra. 1993. Sistema Arrecifal Veracruzano, p. 559-575. In S.I. Salazar-Vallejo \& N.E. González (eds.). Biodiversidad marina y Costera de México. CONABIO y CIQRO, México D.F., México.

Wood, E.J.F. 1968. Dinoflagellates of the Caribean Sea and Adjacent Areas. University of Miami, Coral Glabes, Florida, EEUU.

Zingone, A. 2001. Dinoflagellates (Class Dinoflagellata, Dinophyceae, Phylum Dinophyta) In IOC Taxonomic Reference List of Toxic Algae Intergovernmental Oceanographic Commission of UNESCO, 2002. 